\title{
A Negotiation Optimization Strategy of Collaborative Procurement with Supply Chain Based on Multi-Agent System
}

\author{
Chouyong Chen and Chao Xu \\ Management School, Hangzhou Dianzi University, Hangzhou, Zhejiang 310018, China \\ Correspondence should be addressed to Chao Xu; chaoxu_hdu@163.com
}

Received 13 March 2018; Revised 29 July 2018; Accepted 5 August 2018; Published 26 August 2018

Academic Editor: Luciano Caroprese

Copyright (C) 2018 Chouyong Chen and Chao Xu. This is an open access article distributed under the Creative Commons Attribution License, which permits unrestricted use, distribution, and reproduction in any medium, provided the original work is properly cited.

\begin{abstract}
In the process of collaborative procurement, buyers and suppliers are prone to conflict in cooperation due to differences in needs and preferences. Negotiation is a crucial way to resolve the conflict. Aimed at ameliorating the situations of underdeveloped self-adaptive learning effect of current collaborative procurement negotiation, this paper constructs a negotiation model based on multi-agent system and proposes a negotiation optimization strategy combined with machine learning. It provides a novel perspective for the analysis of intelligent SCM. The experimental results suggest that the proposed strategy improves the success rate of self-adaptive learning and joint utility of agents compared with the strategy of single learning machine, and it achieves win-win cooperation between purchasing enterprise and supplier.
\end{abstract}

\section{Instruction}

Information technology has enabled, and in some cases forced, enterprises to reorient their internal capabilities and to redefine their business models to develop e-commerce techniques. In order to attain timely responsiveness and to proffer higher service level, constructive cooperation among partners in supply chain is critical in any endeavor to ameliorate disruptions and mitigate risks [1]. A small number of successful contemporary associations have transformed from an opportunistic doctrine of cooperation to a synergistic ethos and integrated their supply chain procedures.

The synergism of Cluster Supply Chain (CSC), comprising collaborative manufacture, collaborative procurement, collaborative logistics, and collaborative inventory, is the coupling organizing form between industrial cluster and supply chain. And it helps small and medium-sized enterprise (SME) shorten the transaction cycles and reduce costs. Procurement directly affecting the production and operation is the key link in the development of the whole enterprise. In the fierce market competition, therefore, the purchasing mode gradually shifts from traditional independent purchasing that faces the problems of small quantity discount, low bargaining power, and slow response to customer demand to collaborative procurement.
In this article, we consider a distributed supply chain (SC) in that each member seeks to optimize personal performance and independently plans his business. A large measure of supply chain managements (SCM) have to communicate and negotiate effectively with SC members. In the process of collaborative purchasing, buyers and suppliers are prone to conflict in cooperation due to differences in needs and preferences. Negotiation is a crucial way to resolve the conflict and an effective mechanism for supply chain coordination and cooperation. It has been demonstrated that the information sharing between the buyers and suppliers ensures effective supplier participation and enhances mutual understanding, which contributes to more excellent performance over the rivals [2]. Negotiation is reckoned to be a sound approach for participators to exchange messages, understand other perspectives, and identify new order alternatives based on the information and knowledge learned in the process. And it allows enterprises in the CSC to prevent both self-interest and local optimization of finicky partner, to proceed to the optimization of objectives of all participators and to achieve a win-win situation in SCM.

Former researchers have paid much attention to negotiation problem over the past decade and proposed some salient models. The majority of models primarily use either methods of the improved algorithms or game-theoretic techniques 
as a basis to formulate autonomous negotiation. However, those approaches are considered to be complicated to spread to widespread problem fields due to the uncertainty and complexity in real-world negotiation. This paper ameliorates the negotiation model combining multi-agent system (MAS) with machine learning for further tackling the conflict in cooperation. New model provides a buyer with a method for purchasing a product systematically. And it helps in achieving a win-win cooperation between two sides during the process of collaborative procurement with supply chain as far as feasible.

The remainder of the paper is structured as follows. Section 2 shows literature review. Section 3 recalls some general concepts of key techniques. Section 4 is devoted to a negotiation model of collaborative procurement based on MAS. Section 5 describes a self-adaptive negotiation optimization strategy combined with dynamic selective ensemble learning. Finally, the experiments design, results, and concluding remarks are presented in Sections 6 and 7, respectively.

\section{Literature Review}

Given that research on negotiation of collaborative procurement is new and largely fragmented, it is practically paramount to arouse individuals' attention. Previous studies have, nevertheless, proposed a basic model of supply chains and a negotiation strategy for solving conflicts in consideration of efficiency and cost. A multi-objective cooperative production-distribution planning model was formulated by Jolai et al. [3] applying the fuzzy goal programming approach to maximize the gains of all participators. To discover the optimal solutions of resource allocation, Lin et al. [4] recommended a collaborative negotiation mechanism that was built on price schedules decomposition algorithm. But the popular methods to research the negotiation's conundrum in SCM involve Game Theory and artificial intelligence (AI). Game theorists deem the negotiation as an incomplete, dynamic information game, and attempt to settle the game by offering some predictions on certain conditions [5]. Primary methodological tools of Game Theory are Nash game [6] and Stackelberg game [7], which concentrate on the sequential and simultaneous decision-making of multiple players, respectively. For those relevant analytic modeling studies, the problem is analyzed mostly from a theoretical perspective. Despite being extremely successful in a quantity of situations, the game theoretical approach is considered to be difficult to spread to universal problem fields owing to the uncertainty and complexity in real-world negotiation.

Compared to Game Theory, participants that bargain with consideration of human preference and thoughts could be considerably represented by the agent technology which is a branch of AI [8]. The use of information and communication technology tools, offering the capacities of customer sensitivity, information sharing, and process integration, is observed as the uppermost enabler for this collaborative perception's realization [9]. In computer science, an agent is generally considered as a software entity, which is autonomous to communicate and coordinate with other agents to accomplish its design objectives. Consequently, multi-agent simulation modeling, which originated from AI, is suitable for the conduction of distributed system and has certain advantages in being testable, quantifiable, and efficient. It is superior in expansibility, is easy to configure, and has been widely used in the SCM. Kwon et al. [10] constructed an integrated framework that was based on multi-agent cooperation and casebased reasoning to help address emerging uncertainties. Lin et al. [11] demonstrated a supply chain coordination model of multi-agent and put forward a conflict solution method built on constraint satisfaction algorithm due to the different form of demand. Considering the conflict between businesses caused by the difference of information asymmetry and goals, Behdani et al. [12] developed a negotiation method based on multi-agent in the condition that demand is uncertain. The significance of addressing negotiation mechanisms for collaborative matters is shown by the discussed literatures. The combination of negotiation model and optimization technology is requisite to help negotiators achieve optimal selections.

In order to better promote the agent's self-adaptive negotiation ability, an army of scholars have begun to introduce machine learning into the negotiation. Bayesian Learning estimates the probability distribution of opponent negotiation parameters and preferences and adaptively adjusts the concession strategy [13]. Q-Learning generates the optimal negotiation strategy by calculating the utility cumulative value [14]. Radial Basis Function (RBF) neural network is capable of optimizing the Actor-Critic learning algorithm to predict and amend the concession magnitude of agents [15]. Unfortunately, previous self-adaptive negotiation is built on a single or integrated learning machine to draw the final result [16]. Selective ensemble learning improves the efficiency of general integrated learning machine by eliminating the less accurate ones in sublearning models [17].

This paper is built on our previous work in the field of automated negotiation. In particular, it lays the foundation for accomplishing an experiment to investigate the performance of agent which is operating in the supply chain system and equipped with our negotiation model. The main contribution consists of constructing a negotiation model concerning collaborative procurement based on MAS by analyzing the characteristics of multilateral transact and proposing a negotiation strategy founded on dynamic selective ensemble learning. We exploited supply chain analysis detailedly that was based on agent technology, which detects novel patterns through the improved data mining techniques and provides a new perspective for the analysis of intelligent SCM. Moreover, agent job was led by this association between intelligent agents and machine learning to do faster and better. And the negotiation strategy has also potential for big data decrement and compression.

\section{Methods}

3.1. Machine Learning. Machine learning gradually becomes an irreplaceable method for processing data in the big data era. As an embranchment of AI, it has entered foreland of the mainstream computer science's research that often uses statistical techniques to give agents the ability to learn with data, without being explicitly programmed. Machine learning 
has substantial connections with mathematical optimization, which delivers theory, application domains, and methods to the field. Moreover, it is a popular method practiced to devise complicated models and algorithms for prediction. These analytical models permit researchers to find results and authentic decisions and reveal hidden insights via learning from historical relationships and tendencies in the data.

3.2. K-Means Clustering. K-means clustering, an unsupervised learning, is fundamentally a partitioning method that is utilized to analyze data and treat the data's observations as objects on the basis of locations and distance between diverse input data points. It helps to partition the undisposed objects into mutually exclusive clusters $(\mathrm{K})$ so that objects remain as close as possible to each other within individual cluster but as far as possible from other clusters' objects.

3.3. Support Vector Machine. Support Vector Machine (SVM), introduced by Vapnik, is originated from the theory of structural risk minimization belonging to statistical learning theory. The essential idea of SVM is to map input vectors into a high dimensional feature space and construct the optimal separating hyperplane in this space. SVM tries to minimize an upper bound of the generalization error by maximizing the margin between the test data and the separating hyperplane [18]. It has several merits: (1) A unique hyperplane maximizing the margin of separation between the classes can be discovered by SVM, so it has a good ability of robustness. (2) SVM's power is to use kernel function to transform data from the low dimension space to the high dimension space and create a linear binary classifier. (3) The solving of SVM is a convex programming problem, and its local optimum is selected as the global optimum. In the field of machine learning, models combined with learning algorithms for analyzing and classifying data are represented by SVM.

\section{Negotiation Model of Collaborative Procurement Based on MAS}

One of the most distinguishing advantages of using MAS for SCM is the dynamic supply chain construction via automated negotiation between agents. In the MAS, the coordinator agent is introduced to regulate multiple buyer and seller agents. A distributed negotiation model based on MAS is demonstrated in Figure 1. The model assists enterprises in choosing the most suitable suppliers quickly, efficiently, and economically. The system consists of 3 mutually coordinated agents: CA represents the supplier agent, PA the purchasing enterprise agent of industrial cluster, and MA the broker agent of collaborative purchasing service. Agents participating in the negotiation must register with MA (such as an ecommerce platform) in advance and configure a unique ID. The MA manages various information in the negotiation process and coordinates the communication between the agents. The selection of the supplier is done with the assistance of the MA and repeated negotiation between the PA and the CA (the types of messages used by the agents in the negotiation process are shown in Table 1).
MA: (i) It promptly registers, verifies, and updates information about registered agents. (ii) It duly publishes, forwards, and organizes messages. (iii) It comprehensively utilize real-time environment and enterprise data to evaluate the operation of businesses.

PA: If Reply is received, PA will compare the property values of the products given by the participating CA with accredited ones, and then send Improve to the nonoptimal CA. Subsequently, it selects CA whose values are no less than the threshold as a candidate supplier. If there is no qualified supplier, purchasing enterprise will modify the relevant threshold and renegotiate with all suppliers. Finally, the result opted for is sent to the MA with Selection. After receiving Confirm, if the $\mathrm{CA}$ is found to have objected to the negotiation result, check the modification and resend Improve until no objection occurs.

CA: After monitoring Announce published by the MA, if the requirements of order are met, deliver the Bid to participate in the negotiation. In the event of corresponding values suggested by the PA being acceptable, during Adjust, CA sends a new Bid, or else emits Reject. Eventually, when receiving Result, the selected CA checks the content of the protocol, and if there is no objection, the Accept is fed back. Otherwise, the Refuse is transmitted to point out the problem.

The specific negotiation process is showed in Figure 2.

\section{Self-Adaptive Negotiation Optimization Strategy}

5.1. Negotiation Parameter. Negotiation parameters consisted of four elements which are proposed and explained in Table 2.

$$
\mathrm{NM}=\{\mathrm{A}, \mathrm{P}, \mathrm{w}, \mathrm{U}\}
$$

\subsection{Concessional Learning Based on Dynamic Selective} Ensemble of SVM. According to the current negotiation issues, the nearest neighbor sample set is used as the training sample to evaluate the performance of each submodel and select the better ones. In the negotiation, K-means algorithm is adopted for each negotiation issue, and the $\mathrm{k}$ sample subsets are found as the training datasets. And the Support Vector Machine (SVM) is used to learn the concession amplitude in each evaluation sample. Taking root-meansquare error (RMSE) as the evaluation criterion, we eliminate some submodels with poor performance. The combination weight is calculated and the final dynamic selective SVM model is established.

(1) K-means algorithm generates evaluation datasets. $P_{q}$ is negotiation sequence to be predicted and its number of the nearest neighbor sample in the data set $P_{L}$ is $\mathrm{k}$, and the first $\mathrm{k}$ samples $P_{\mathrm{k}}$ can be got by calculating the Euclidean Distance $P_{D}$ between $P_{q}$ and the sample points $P_{\mathrm{i}}$.

$$
P_{D}\left(P_{q}, P_{i}\right)=\sqrt{\sum_{i \in L}\left(P_{q}-P_{i}\right)^{2}}
$$

(2) Input sample set $P_{\mathrm{k}}$, and estimate concession amplitude with SVM. Assume that negotiation values of $A_{C}$ and $A_{P}$ in round $\mathrm{t}$ and issue $\mathrm{j}$ are denoted as $P_{t}^{C}$ and $P_{t}^{P}$, respectively, 


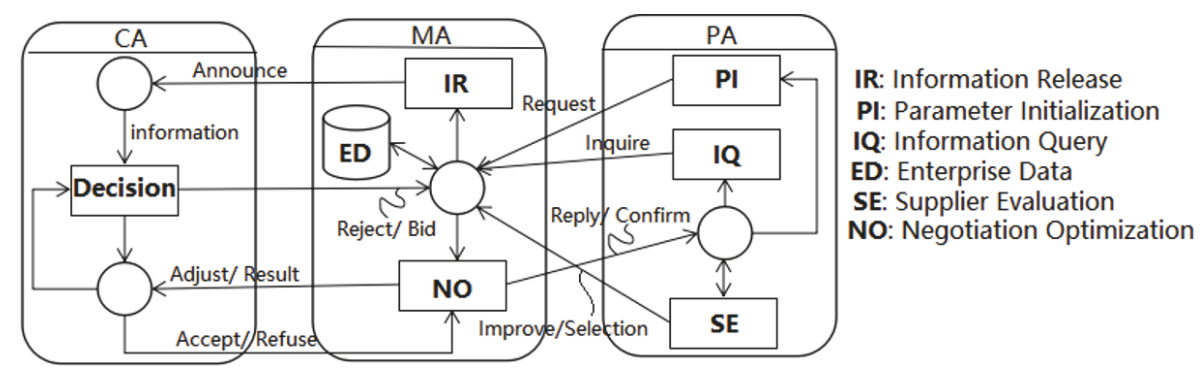

FIGURE 1: MAS negotiation model of collaborative procurement.

TABLE 1: Instructions of related messages.

\begin{tabular}{|c|c|c|}
\hline Agent & Message & \\
\hline \multirow{7}{*}{ MA } & Announce & $\mathrm{F}$ \\
\hline & Adjust & \\
\hline & Result & Inf \\
\hline & Reply & \\
\hline & Confirm & Transforn \\
\hline & Request & \\
\hline & Inquire & \\
\hline \multirow[t]{3}{*}{ PA } & Improve & \\
\hline & Selection & \\
\hline & Reject & \\
\hline \multirow[t]{3}{*}{ CA } & Bid & Give informa \\
\hline & Accept & \\
\hline & Refuse & \\
\hline \multicolumn{3}{|c|}{ TABLE 2: Instructions of negotiation parameters. } \\
\hline Parameters & Instructic & \\
\hline A & $A_{C}$ represents Supplier, $A_{\mathrm{P}} \mathrm{I}$ & cluster buyer \\
\hline $\mathrm{P}$ & Issue value of ne & \\
\hline $\mathrm{w}$ & Weight vector of & \\
\hline $\mathrm{U}$ & Utility value of & \\
\hline
\end{tabular}

and $\Delta D_{t}$ is the negotiation difference between $A_{C}$ and $A_{P}$ obtained by (3). The average concession amplitudes of $A_{C}, A_{P}$ for the first t rounds are $\bar{C}_{t}^{C}, \bar{C}_{t}^{P}$. As inputs to the SVM, t, $\Delta D_{t}$, $\bar{C}_{t}^{C}$, and $\bar{C}_{t}^{P}$ are mapped to the high dimensional space using the Radial Basis Function $H_{t}=\left(\varphi(t), \varphi\left(\Delta D_{t}\right), \varphi\left(\vec{C}_{t}^{C}\right), \varphi\left(\bar{C}_{t}^{P}\right)\right)$. $C_{t+1}^{P}$ is the output variable of the linear regression function obtained by (5).

$$
\begin{gathered}
\Delta D_{t}=\left|P_{t}^{C}-P_{t}^{P}\right| \\
\bar{C}_{t}^{P}=\sum_{i=2}^{t} \frac{\Delta D_{i}}{P_{i-1}^{P}}
\end{gathered}
$$

$$
C_{t+1}^{P}=w^{T} *\left(\varphi(t), \varphi\left(\Delta D_{t}\right), \varphi\left(\bar{C}_{t}^{P}\right), \varphi\left(\bar{C}_{t}^{C}\right)\right)+b
$$

where $w^{T}$ is the weight vector of 4 input variables and $b$ is a offset value.

The error $\varepsilon$ between predicted value y and function value $C_{t+1}^{P}$ could be calculated by (6). If the error $\varepsilon$ is regarded as an error-free fitting, then we can get the nonlinear regression function as (7) of the concession amplitude $C_{t+1}^{P}$ of the opponent in round $t+1$. After the equivalent substitution, we can get the final regression function as (8).

$$
\begin{gathered}
\max \left\{0,\left|y-C_{t+1}^{P}\right|-\varepsilon\right\} \\
C_{\mathrm{t}+1}^{P}=\sum_{j=1}^{n}\left(a_{j}-a_{\mathrm{j}}^{\prime}\right) K\left(H_{t}, H_{t-1}\right)+b \\
C_{\mathrm{t}+1}^{P}=\sum_{j=1}^{n} a_{j} \exp \left\{-\frac{\left\|H_{t}-H_{t-1}\right\|^{2}}{\sigma^{2}}\right\}+b
\end{gathered}
$$

where $a_{j}\left(a_{j}>0\right)$ is a Lagrange multiplier, identified by SVM training. Similarly, $C_{t+1}^{C}$ is the predictive concession amplitude value of $A_{C}$ in round $\mathrm{t}+1$. 


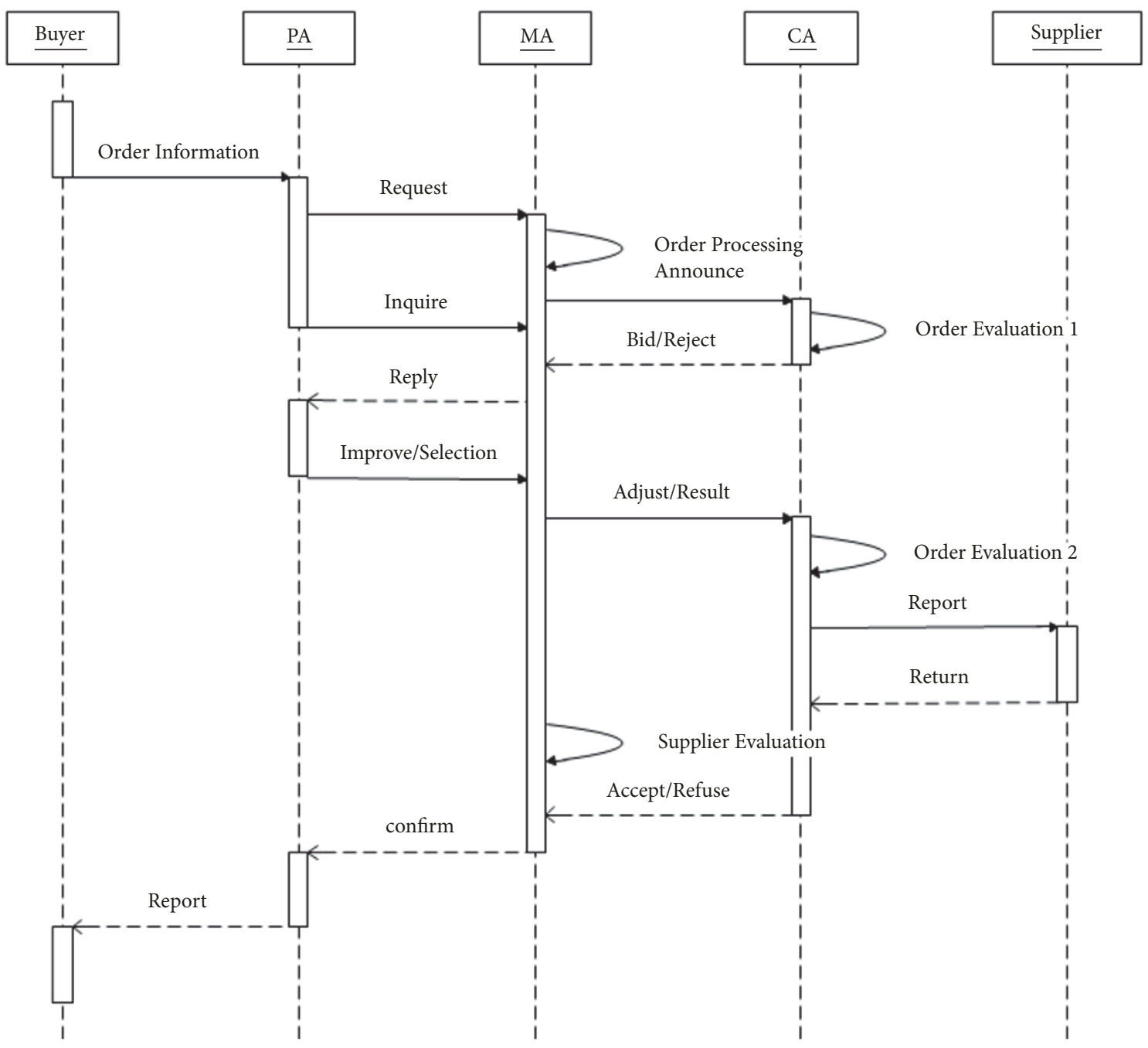

FIGURE 2: MAS sequence diagram of negotiation model.

(3) Using the RMSE as a filter criterion as (9), we select the corresponding first $\bar{k}$ sublearning machines.

$$
E_{i j}=\sqrt{\frac{\sum_{i=1}^{k}\left(\widetilde{c}_{i j}-C_{i j}\right)^{2}}{k}}
$$

where $\widetilde{c}_{i j}$ is the next predictive concession value in issue $\mathrm{j}$ of sublearning machine $\mathrm{i}$ and $C_{i j}$ means the actual concession amplitude.

(4) Calculate the combined weight of each submodel. According to the RMSE value $E_{i j}$ of the $i$-th submodel, the weight of the submodel is obtained.

$$
\alpha_{i}=\frac{\left(1 / E_{i j}^{2}\right)}{\left(\sum_{i=1}^{\bar{k}}\left(1 / E_{i j}^{2}\right)\right)}
$$

When all the k sublearning machines are successfully trained, select the $\bar{k}$ sublearning models with the smallest error. Input the actual concession $C_{i j}$, and then get the output of ultimate concession about issue $j$ in the round $t+1$.

$$
C_{t+1, j}^{C / P}=\sum_{i=1}^{\bar{k}} \alpha_{i} C_{i j}
$$

5.3. Utility Optimization. Taking $A_{P}$ as an example, the utility difference of sequential negotiations is used to decide whether to stop the current consultation. $C_{t+1, j}^{P}$ means a predictive concession value about issue $\mathrm{j}$ in round $\mathrm{t}+1 . P_{t, \mathrm{j}}^{P}$ is an actual value of buyer $A_{P}$ about issue $\mathrm{j}$ in round $\mathrm{t}$.

$$
\begin{gathered}
U_{\mathrm{t}}=\sum_{j=1}^{n} w_{j} P_{t, j}^{P} \\
P_{t+1, j}^{P}=P_{t, j}^{P}+C_{t+1, j}^{P}
\end{gathered}
$$

The error between the predictive utility value in round $t+1$ and actual utility value in round $\mathrm{t}$ can be calculated by coordinating equations (12) and (13). While $\Delta U_{t+1, t}>0$, the 


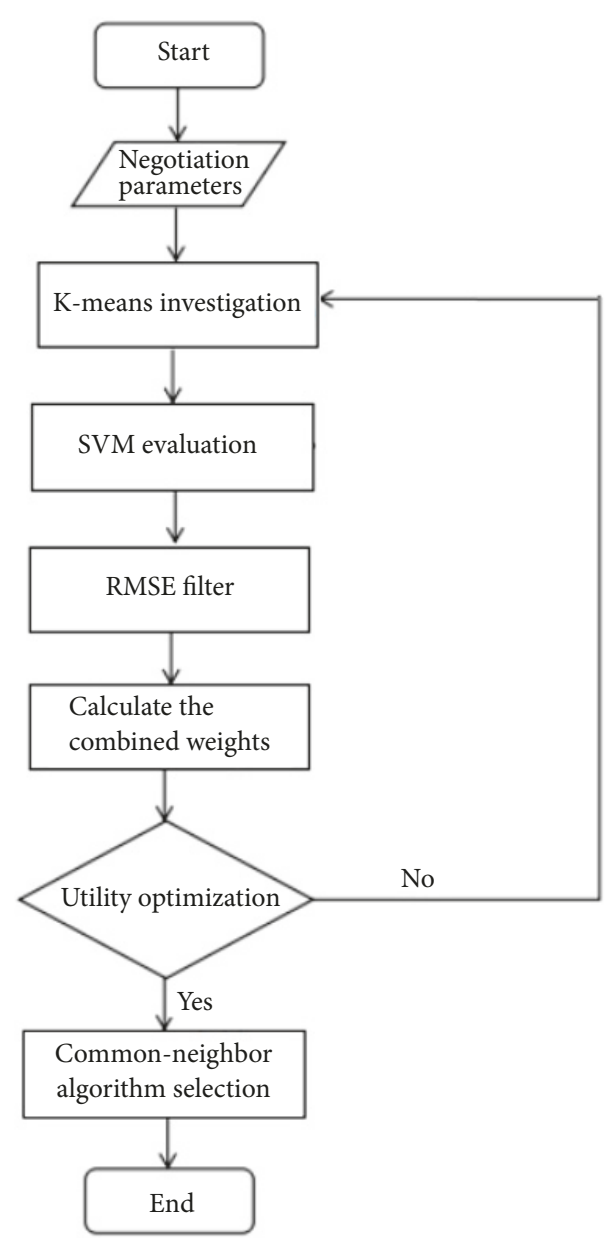

FIGURE 3: Flowchart of Self-adaptive negotiation optimization strategy.

utility of concession has not been maximized; it will increase. Conversely, end the concession.

5.4. Selection of the Most Appropriate Partner. After the negotiation, the common-neighbor algorithm [19] is applied to compute the similarity of the issues, and $A_{\mathrm{P}}$ choose more suitable partners according to the similarity.

$$
S_{P, C}=\left(1+e^{-D_{P, C} / 2}\right) *\left\|I_{P} \cap I_{C}\right\|
$$

$D_{P, C}$ means the total issue difference between $A_{\mathrm{P}}$ and $A_{C} \cdot\left\|I_{P} \cap I_{C}\right\|$ is the quantity of accredited issue after the negotiation.

Procedures are as follows (see Figure 3). First, K-means search was adopted to generate sample sets. Second, the SVM was used to learn the concession amplitude in each evaluation sample and then eliminated the poor performance of sublearning model with RMSE and calculated the combined weight and the final dynamic selective SVM model was established. Third, the utility function was used to decide whether to terminate the negotiation. Finally, the most appropriate partner was selected on the basis of issues' similarity calculated with common-neighbor algorithm.
Furthermore, the self-adaptive negotiation optimization strategy is also suitable for complicated problems of big data in massively parallel environments. The complexity of big data could be decreased by data processing algorithms' application.

\section{Simulation Example}

Relying on modern logistics network system, Yiwu has become the largest small commodity distribution center in the world. The merchandise is sold to Europe, America, the Middle East, and South Asia and other regions. Yiwu market now has more than 4.3 million square meters of business area, 63 thousand operators, and more than 400 thousand kinds of products. In 2016, the trading volume of commodity markets reached 373 billion $\mathrm{RMB}$ and the total export-import volume extended to 223 billion RMB (Yiwu China Commodities City Group Official Website 2017). Yiwu Global Purchasing (www.yiwuok.com) as an e-commerce platform contributed $60 \%$ of the first value. The key link of supply chain synergism is to utilize e-commerce platform services to develop a healthy relationship of trust among partners and establish an effective mechanism for information collaboration. This paper takes Yiwu Small Commodity Industry Cluster (SCIC) as an instance and grabs five main parameters: product price, quantity, delivery time, warranty time, and defective rate as the negotiation issue. The effectiveness of self-adaptive Integrated Optimization Strategy (IOS) is verified by using Matlab R2014a, which is compared with the General Learning Strategies (GLS) based on single SVM.

According to the historical data analysis of electric appliances industry in Yiwu SCIC, the supplier cares more about price, quantity, and delivery time, while concentrating less on warranty time and defective rate. The purchasing enterprise is a little bit different; they focus on defective rate rather than warranty time, demonstrated detailedly in Table 3. Initial experimental datasets could be extracted from Dataverse repository. The whole examinations were performed on a laptop (4 GB of RAM that operated under Windows 10 desktop, Intel core i3 CPU @ 2.54 GHz). In addition, we selected the open source libraries, VLFeat for K-means clustering and LIBSVM for SVM algorithm, with excellent interfaces in Matlab for ease of use. To get the generation of optimal solutions, the experimental time is limited to 2 minutes.

A separating hyperplane of datasets illustrated by the IOS is exhibited in Figure 4. In place of the smaller margin, the hyperplane creates sheltered subregions to make most examples with identical class label drop on the same side of the decision boundary. And subregions are produced by decision boundary with diverse piecewise shapes, such as jutting out as peninsulas that are virtually surrounded by the antagonists. The misclassifications might comprise some stray examples submerged in the opponents. As the crucial target of sustaining the native class' membership, the IOS eliminates the stray examples - those characterized as black solid symbols-from the hyperplane. As mentioned above, we are working on the assumption that the margin shrinkage is a price to trade off with the misclassification decrease in the practice stage. 
TABLE 3: Intervals and weights of negotiation issue.

\begin{tabular}{lcccc}
\hline Parameters & Intervals of supplier's issue & Intervals of purchaser's issue & Weight vector of supplier & Weight vector of purchaser \\
\hline Price/Yuan & {$[100,150]$} & {$[100,130]$} & 0.40 & 0.35 \\
Quantity & {$[800,1000]$} & {$[850,1200]$} & 0.25 & 0.30 \\
Delivery time/Month & {$[1.5,2]$} & {$[1,2]$} & 0.20 & 0.20 \\
Warranty time/Month & {$[12,18]$} & {$[15,24]$} & 0.10 & 0.05 \\
Defective rate/\% & {$[80,95]$} & {$[90,95]$} & 0.05 & 0.10 \\
\hline
\end{tabular}

TABLE 4: Error rate (\%) comparison of experimental results.

\begin{tabular}{lccccc}
\hline Strategy & Min error & Max error & Median error & Average error & Standard Deviation \\
\hline GLS & 2.8 & 32.1 & 14.2 & 15.86 & 8.37 \\
IOS & 3.2 & 26.1 & 10.1 & 11.97 & 6.05 \\
\hline
\end{tabular}

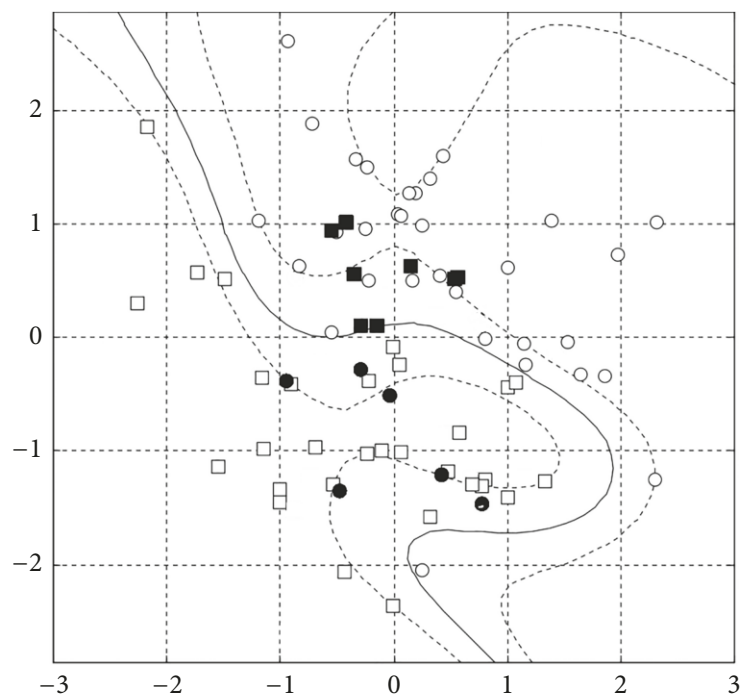

FIGURE 4: A separating hyperplane depicted by the IOS.

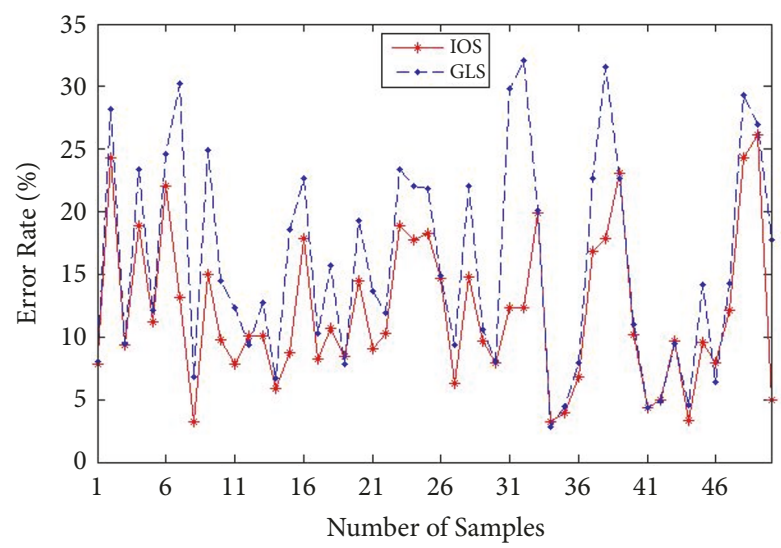

FIGURE 5: Simulation results of error rate of 2 strategies.

50 couples are selected in the experiment to predict the margin of opponent concessions by comparing two strategies. According to Figure 5, we can draw the conclusion that in most cases this IOS infers lower error rate than the ordinary

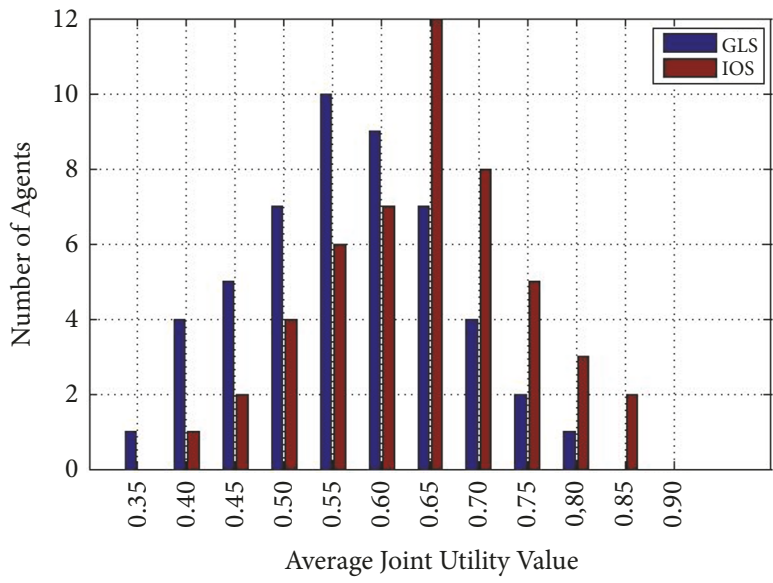

FIgURE 6: The comparison of joint utility and successful agent.

single SVM. Additionally, the basic descriptive statistics of the data is provided in Table 4. The average error of IOS for all 50 objects is $11.97 \%$ with a standard deviation of $6.05 \%$. It could be seen that the IOS outperforms the GLS in four vital error measures. The max error is $6.0 \%$ lower, the median error is $4.1 \%$ lower, the average error is $3.89 \%$ lower, and standard deviation is $2.32 \%$ lower than the GLS, respectively.

In Figure 6, the average joint utility value founded by IOS is mainly concentrated in $[0.50,0.75]$, while another value is mainly concentrated in $[0.40,0.70]$. The total average joint utility of the former is 0.641 , and $60 \%$ of agents are higher than that value. Nevertheless, the numbers of the latter calculated severally are 0.565 and $46 \%$. Distinctly, the strategy proposed by this paper is superior to GLS in both the amounts of successful agents and joint utility value.

\section{Conclusions}

Previous studies proposed a number of basic supply chain models which are difficult to spread to universal problem fields owing to the uncertainty and complexity in realworld negotiation. The most fascinating modern application of ensemble systems lies in processing high dimensional, complex, and big data that cannot be analyzed efficiently 
by single-model methods. To better solve the conflict in negotiation, this paper has discussed the negotiation problem of collaborative procurement operating on MAS model with a negotiation optimization strategy. We exploited supply chain analysis minutely based on agent technology and machine learning, which provides a new perspective for the analysis of intelligent SCM. Apparently, we perceive that the negotiation and learning are key aspects in the system performance by the simulation of the proposed MAS model for the procurement management of CSC. The agents have symmetric preferences, complicating the negotiation. However, the learning helped each one acquire the ultimate strategy choice. The experimental results show that the IOS based on dynamic selective ensemble SVM can reduce the error rate and elevate the joint utility, compared with GLS of the ordinary single learning machine. The test reveals that the model plays a key role in negotiation issue inside the intelligent SCM, and the agent negotiation performance and efficiency can be enhanced via the combination of the improved data mining techniques.

The procurement management of supply chain involves fabrication, inventory, distribution, and other issues, and the supply chain needs collaboration of upstream and downstream enterprises to achieve a synergistic, dynamic, and timely supply-production-marketing operation mode. Future research will focus on the resolution of conflict in selfadaptive negotiation to further improve the intelligent level of supply chain.

\section{Data Availability}

The datasets analyzed during the current study are available in Dataverse repository: https://dataverse.harvard.edu/dataset .xhtml? persistentId=doi\%3A10.7910\%2FDVN\%2FVT2AQJ\& version $=$ DRAFT.

\section{Conflicts of Interest}

The authors declare that there are no conflicts of interest regarding the publication of this paper.

\section{Acknowledgments}

This work is supported by NSFC-Zhejiang Joint Fund for the Integration of Industrialization and Informatization (U1509220).

\section{References}

[1] J. Hallikas, I. Karvonen, U. Pulkkinen, V.-M. Virolainen, and M. Tuominen, "Risk management processes in supplier networks," International Journal of Production Economics, vol. 90, no. 1, pp. 47-58, 2004.

[2] Y. Zhang, L. Wang, and J. Gao, "Supplier collaboration and speed-to-market of new products: the mediating and moderating effects," Journal of Intelligent Manufacturing, vol. 28, no. 3, pp. 805-818, 2017.

[3] F. Jolai, J. Razmi, and N. K. Rostami, "A fuzzy goal programming and meta heuristic algorithms for solving integrated production: distribution planning problem," Central European Journal of Operations Research, vol. 19, no. 4, pp. 547-569, 2011.
[4] Y.-I. Lin, Y.-W. Chou, J.-Y. Shiau, and C.-H. Chu, "Multi-agent negotiation based on price schedules algorithm for distributed collaborative design," Journal of Intelligent Manufacturing, vol. 24, no. 3, pp. 545-557, 2013.

[5] K. Govindan, A. Diabat, and M. N. Popiuc, "Contract analysis: a performance measures and profit evaluation within twoechelon supply chains," Computers \& Industrial Engineering, vol. 63, no. 1, pp. 58-74, 2012.

[6] M. Leng and M. Parlar, "Game theoretic applications in supply chain management: a review," Infor Information Systems \& Operational Research, vol. 43, no. 3, pp. 187-220, 2005.

[7] J.-C. Hennet and S. Mahjoub, "Toward the fair sharing of profit in a supply network formation," International Journal of Production Economics, vol. 127, no. 1, pp. 112-120, 2010.

[8] N. C. Karunatillake, N. R. Jennings, I. Rahwan, and P. McBurney, "Dialogue games that agents play within a society," Artificial Intelligence, vol. 173, no. 9-10, pp. 935-981, 2009.

[9] Y. Wu and J. Angelis, "Achieving agility of supply chain management through information technology applications," International Federation for Information Processing, vol. 246, pp. 245253, 2007.

[10] O. Kwon, G. P. Im, and K. C. Lee, "MACE-SCM: a multi-agent and case-based reasoning collaboration mechanism for supply chain management under supply and demand uncertainties," Expert Systems with Applications, vol. 33, no. 3, pp. 690-705, 2007.

[11] F.-R. Lin and Y.-Y. Lin, "Integrating multi-agent negotiation to resolve constraints in fulfilling supply chain orders," Electronic Commerce Research and Applications, vol. 5, no. 4, pp. 313-322, 2006.

[12] B. Behdani, A. Adhitya, Z. Lukszo, and R. Srinivasan, "Negotiation-based approach for order acceptance in a multiplant specialty chemical manufacturing enterprise," Industrial \& Engineering Chemistry Research, vol. 50, no. 9, pp. 5086-5098, 2011.

[13] J. Zhang, F. Ren, and M. Zhang, "Bayesian-based preference prediction in bilateral multi-issue negotiation between intelligent agents," Knowledge-Based Systems, vol. 84, pp. 108-120, 2015.

[14] L. Chen, H. Dong, and Y. Zhou, "A reinforcement learning optimized negotiation method based on mediator agent," Expert Systems with Applications, vol. 41, no. 16, pp. 7630-7640, 2014.

[15] Z. Ma, C. Wang, Y. Niu, X. Wang, and L. Shen, "A saliencybased reinforcement learning approach for a UAV to avoid flying obstacles," Robotics and Autonomous Systems, vol. 100, pp. 108-118, 2018.

[16] J. Heinermann and O. Kramer, "Machine learning ensembles for wind power prediction," Journal of Renewable Energy, vol. 89, pp. 671-679, 2016.

[17] Y. Liu, B. He, D. Dong et al., "Particle swarm optimization based selective ensemble of online sequential extreme learning machine," Mathematical Problems in Engineering, vol. 2015, Article ID 504120, 10 pages, 2015.

[18] N. B. Peng, Y. X. Zhang, and Y. H. Zhao, "A SVM-kNN method for quasar-star classification," Science China Physics, Mechanics \& Astronomy, vol. 56, no. 6, pp. 1227-1234, 2013.

[19] Y. H. He, D. B. Chen et al., "Similarity algorithm based on users common neighbors and grade information," Computer Science, vol. 37, no. 9, pp. 184-186, 2010. 


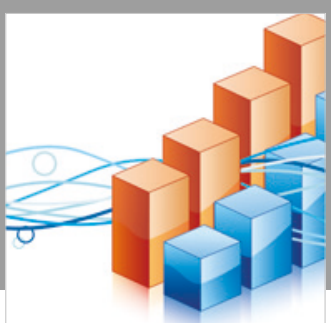

Advances in

Operations Research

\section{-n-m}
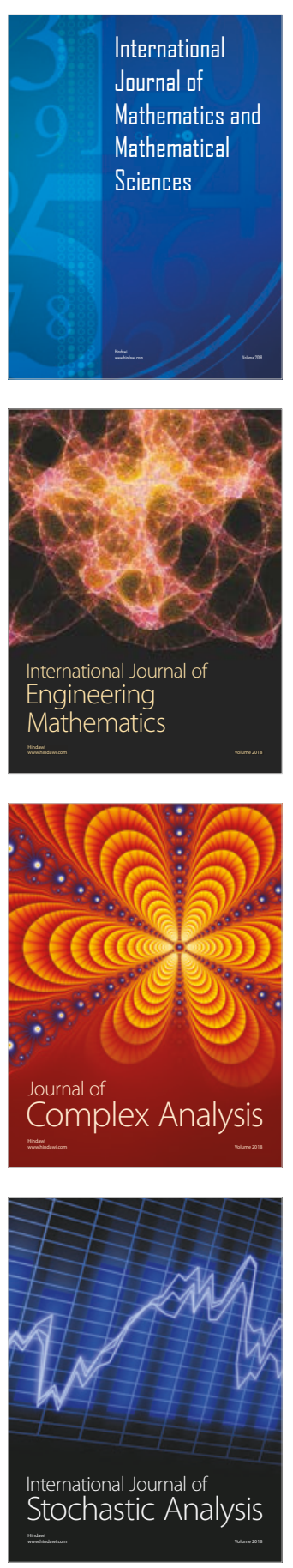
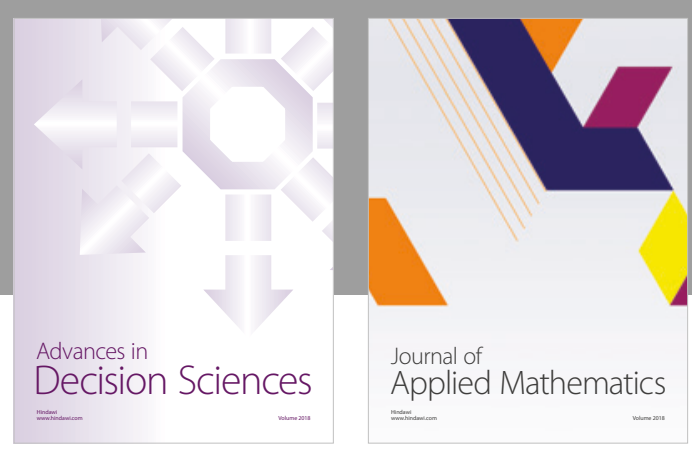

Journal of

Applied Mathematics
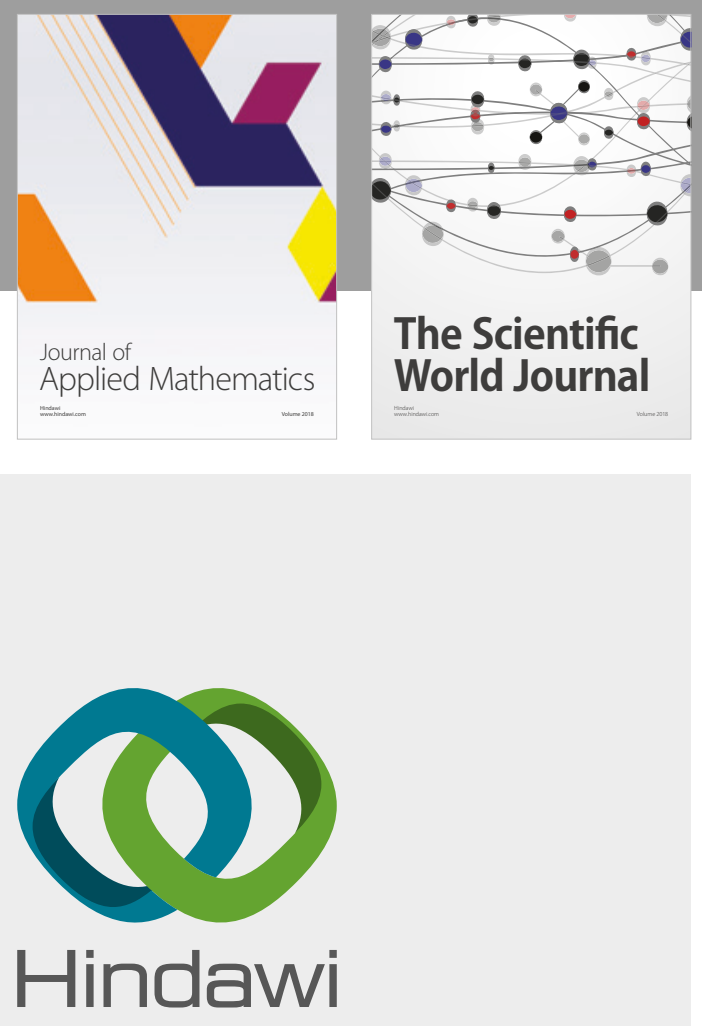

Submit your manuscripts at

www.hindawi.com

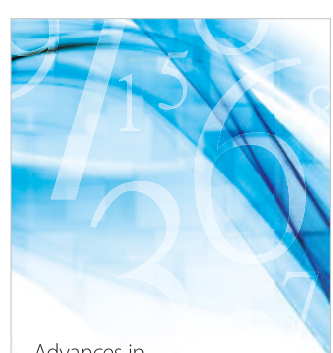

Advances in
Numerical Analysis
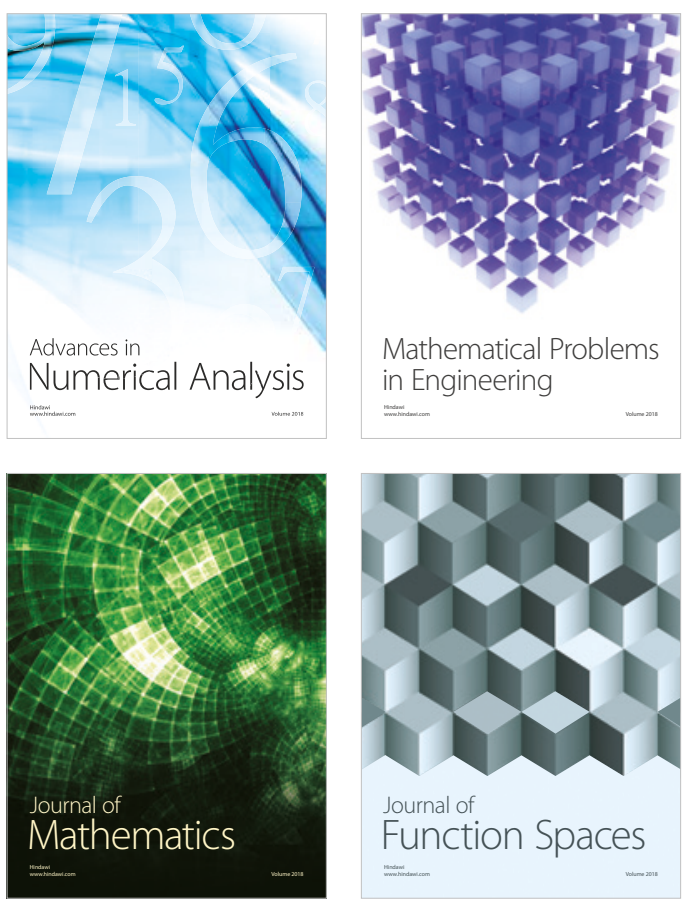

Mathematical Problems in Engineering

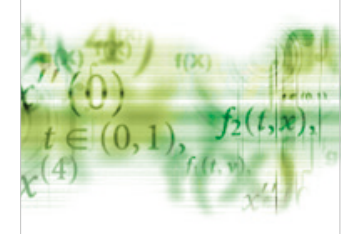

International Journal of

Differential Equations

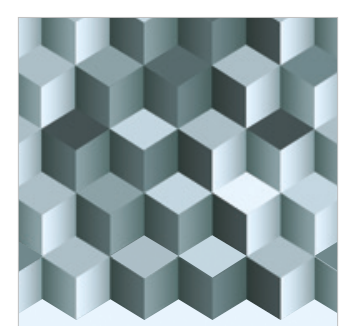

Journal of

Function Spaces

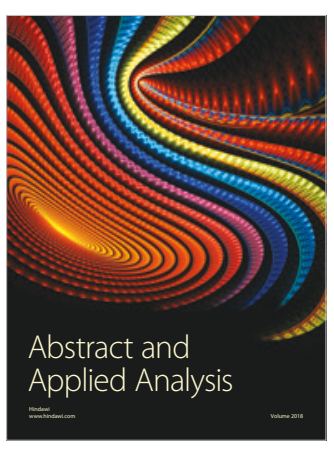

The Scientific

World Journal

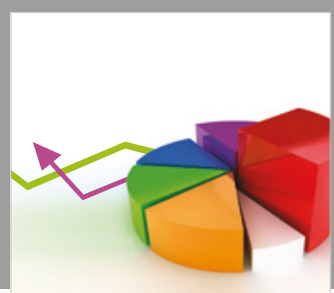

Journal of

Probability and Statistics
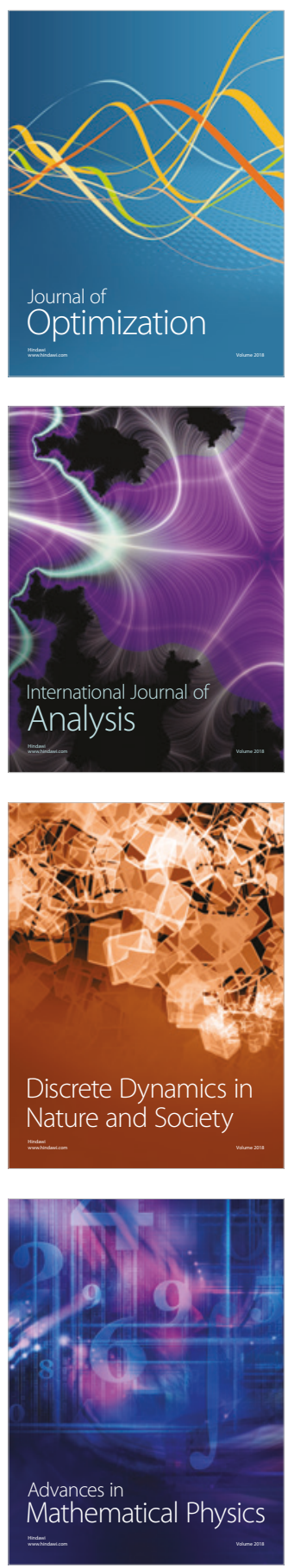